\title{
Neuronal maturation reduces the type I IFN response to orthobunyavirus infection and leads to increased apoptosis of human neurons
}

Clayton W. Winkler ${ }^{1 *}$ (D, Tyson A. Woods' ${ }^{1}$ Bradley R. Groveman ${ }^{1}$, Aaron B. Carmody², Emily E. Speranza ${ }^{3}$, Craig A. Martens ${ }^{2}$, Sonja M. Best ${ }^{3}$, Cathryn L. Haigh ${ }^{1+}$ and Karin E. Peterson ${ }^{1+}$

\begin{abstract}
Background: La Crosse virus (LACV) is the leading cause of pediatric arboviral encephalitis in the USA. LACV encephalitis can result in learning and memory deficits, which may be due to infection and apoptosis of neurons in the brain. Despite neurons being the primary cell infected in the brain by LACV, little is known about neuronal responses to infection.

Methods: Human cerebral organoids (COs), which contain a spectrum of developing neurons, were used to examine neuronal responses to LACV. Plaque assay and quantitative reverse transcription (qRT) PCR were used to determine the susceptibility of COs to LACV infection. Immunohistochemistry, flow cytometry, and single-cell transcriptomics were used to determine specific neuronal subpopulation responses to the virus.

Results: Overall, LACV readily infected COs causing reduced cell viability and increased apoptosis. However, it was determined that neurons at different stages of development had distinct responses to LACV. Both neural progenitors and committed neurons were infected with LACV, however, committed neurons underwent apoptosis at a higher rate. Transcriptomic analysis showed that committed neurons expressed fewer interferon (IFN)stimulated genes (ISGS) and genes involved IFN signaling in response to infection compared to neural progenitors. Furthermore, induction of interferon signaling in LACV-infected COs by application of recombinant IFN enhanced cell viability.

Conclusions: These findings indicate that neuronal maturation increases the susceptibility of neurons to LACVinduced apoptosis. This susceptibility is likely due, at least in part, to mature neurons being less responsive to virusinduced IFN as evidenced by their poor ISG response to LACV. Furthermore, exogenous administration of recombinant IFN to LACV COs rescued cellular viability suggesting that increased IFN signaling is overall protective in this complex neural tissue. Together these findings indicate that induction of IFN signaling in developing neurons is an important deciding factor in virus-induced cell death.
\end{abstract}

Keywords: Orthobunyavirus, Encephalitis, Human cerebral organoids, Neurogenesis, Neuron, Type I interferon, Apoptosis, Single-cell transcriptomics

\footnotetext{
* Correspondence: winklercw@niaid.nih.gov

Cathryn L. Haigh and Karin E. Peterson reserve the right to list themselves as

Senior Author when referencing this manuscript.

${ }^{1}$ Laboratory of Persistent Viral Diseases, Rocky Mountain Laboratories,

National Institute of Allergy and Infectious Diseases, National Institutes of

Health, 903 S. 4th Street, Hamilton, MT 59840, USA

Full list of author information is available at the end of the article
}

(c) The Author(s). 2019 Open Access This article is distributed under the terms of the Creative Commons Attribution 4.0 International License (http://creativecommons.org/licenses/by/4.0/), which permits unrestricted use, distribution, and reproduction in any medium, provided you give appropriate credit to the original author(s) and the source, provide a link to the Creative Commons license, and indicate if changes were made. The Creative Commons Public Domain Dedication waiver (http://creativecommons.org/publicdomain/zero/1.0/) applies to the data made available in this article, unless otherwise stated. 


\section{Background}

La Crosse virus (LACV) is an arthropod-borne orthobunyavirus endemic to North America [1]. It was first isolated from the brain of a patient that died of pediatric encephalitis in 1960 [2] and is currently the leading cause of pediatric encephalitis in the USA [3]. Symptoms include headache, fever, and vomiting progressing to lethargy, behavioral changes, and/or brief seizures until resolution 7-8 days after onset [4-6]. In severe cases, neurological symptoms present more quickly and can progress to deep coma. Of symptomatic cases, 50\% develop seizures with status epilepticus occurring in 10$15 \%$. The case fatality rate is low at $\sim 1 \%$, however, longterm sequelae can occur. One study demonstrated abnormal behaviors and EEG readings in $15 \%$ of patients 1-5 years following hospital discharge [7]. Another study reported $32 \%$ of patients with severe LACV encephalitis performed lower than expected on intelligence testing administered 12-18 months post-hospitalization [5] suggesting long-term learning impairment. Despite the clinical importance of this virus, little is known about its pathogenesis in humans.

Several histopathological studies from post-mortem and biopsied human tissues have defined hallmarks of LACV encephalitis that include infection, damage, and degeneration of neurons [2, 4]. However, these studies failed to define whether specific developing neuronal populations were infected or whether infection and/or death of neuronal cells is maturation-dependent. Originally, cultured post-mitotic neurons were found to be more resistant to LACV-induced apoptosis than undifferentiated progenitor cells [8] leading to the dogma that mature adult neurons are resistant to LACV-induced cell death. However, a recent study using human neuronal/ astrocyte co-cultures suggests that differentiated neurons could be infected with LACV and undergo cell death [9]. To directly examine if neuronal maturity affects the ability of LACV to infect and/or induce apoptosis of neurons requires a system with neurons at different stages of maturity. This is particularly important for LACV infection, as LACV primarily causes disease in young children, with the main age range of 4 to 11 , a time point where the brain is still undergoing active neurogenesis [10]. Furthermore, one of the clinical signs associated with LACV infection is learning and memory impairment, processes that are thought to involve hippocampal neurogenesis $[11,12]$. Thus, understanding how LACV may impact neurons at different stages of maturity is critical for understanding disease pathogenesis.

Induced pluripotent stem cell (iPSC)-derived human cerebral organoids (COs) are a highly useful tool in examining virus infection of neurons in that they contain a spectrum of developing neurons within the same complex, interconnected tissue. They exhibit a similar inside-out patterning and regional organization as a developing human brain [13-15], with ventricular zone-like regions populated by SOX2 expressing neural progenitors that give rise to Doublecortin (DCX) expressing immature neurons. These immature neurons are committed to the neuronal lineage [16] and migrate radially away from the neurogenic regions to populate and integrate into the tissue as Class III $\beta$-tubulin ( $\beta$ III-tubulin) expressing mature neurons $[17,18]$. Thus, COs are a unique model in which to study LACV pathogenesis as they contain neurons at varying stages of development and connectivity. In this study, we utilize COs to model viral tropism in neuronal cells at various developmental stages and determine if the developmental stage of neurons influences virus-induced cell death. Additionally, we analyze differences in gene expression between neuronal populations to determine if neuronal maturity affects the response to viral infection.

\section{Methods \\ iPSC-derived human cerebral organoid generation and maintenance}

Cerebral organoids (COs) were generated using the protocol and media formulations described in Lancaster and Knoblich [17], with the following minor variations. Source tissue was KYOU-DXR0109B human-induced pluripotent stem cells (iPSC; ATCC). Embryoid bodies (EBs) were generated using one $25 \mathrm{~cm}^{2}$ flask of cells to seed a 96-well low-adhesion U-well plate. EBs were transferred into neural induction medium between days 4 and 6 depending upon morphology. Organoids were Matrigel embedded on day 3 or 4 post neural induction. Four days after embedding, organoids were transferred into an agitated culture in upright $25 \mathrm{~cm}^{2}$ low-adhesion flasks on an orbital shaker (Heathrow Scientific) at 85 rpm. Media was changed 2-3 times per week until 21 days post neural induction when COs were plated individually into low-adhesion 24 well plates for infection. The agitated culture was maintained, and media completely changed/collected daily.

\section{Virus stocks and infection}

LACV stocks were generated as previously described [19]. The virus for $\mathrm{CO}$ infection was diluted to either $10^{3}$ or $10^{1}$ plaque-forming units (PFU) in $500 \mu \mathrm{l}$ of medium. Medium from wells containing COs was removed and replaced with a virus-containing medium for $2 \mathrm{~h}$ in the incubator while shaking. After infection, wells containing COs were washed and refilled with $1 \mathrm{ml}$ fresh medium.

\section{Treatment of COs with recombinant IFN}

COs were infected and maintain in culture as described above except that beginning at $1 \mathrm{dpi}$ (day post-infection), recombinant human IFN $\beta 1$ or IFN $\alpha 2$ and IFN $\alpha 4$ (PBL 
Assay Science) or vehicle $(1 \times$ PBS with $0.1 \%$ fetal bovine serum) was added to each daily media change. All recombinant IFNs were used at a dose of $1000 \mathrm{U} / \mathrm{ml}$. An equivalent volume of vehicle solution was used for control samples.

\section{RNA isolation and quantitative reverse transcription (qRT) PCR from COs and media supernatant}

At indicated time points, either whole or half COs or cell culture medium was isolated, flash-frozen, and stored at $-80^{\circ} \mathrm{C}$. COs were dissociated by incubation in $0.5 \mathrm{ml}$ of Corning $^{\mathrm{mm}}$ Cell Recovery Solution for $1 \mathrm{~h}$ at $4^{\circ} \mathrm{C}$. Samples were washed 3 times with $1 \times$ PBS by pelleting at $500 \mathrm{~g}$ and then placed into ZR RNA buffer for RNA isolation and clean up according to the manufacturer's protocols (Zymo Research). Viral RNA was isolated from CO culture supernatants using the Quick-RNAViral RNA Kit (Zymo Research) according to the manufacturer's protocols. Analysis of mRNA from COs was performed as previously described [20]. Viral RNA in culture media was determined by fitting sample $C_{T}$ values to a standard curve obtained from a linear regression fit from 10-fold dilutions of spiked cell culture media containing known virus PFUs. Primers used: hGapdh.2-707F 5'-TCGTGGAAGGACTC ATGACC-3', hGapdh.2-818R 5'-ATGATGTTCTGGAG AGCCCC-3' and LACV.2-552F 5'-ATTCTACCCG CTGACCATTG-3', LACV.2-650R5'-GTGAGAGTGC CATAGCGTTG-3'.

\section{Replication kinetics}

COs were infected with LACV as described above and culture supernatants were harvested at 1-6 dpi. Supernatants were titered as described previously [21].

\section{Cell viability assay}

Indicated COs were assayed daily for cell viability using PrestoBlue $^{\mathrm{Tm}}$ (Molecular Probes). Daily, culture medium was removed from each $\mathrm{CO}$-containing well and replaced with $0.5 \mathrm{ml}$ of $1 \times$ PrestoBlue $^{\text {tw }}$ reagent in medium for a 30 min incubation. Samples and controls were read in triplicate using fluorescence excitation at $560 \mathrm{~nm}$ and emission at $590 \mathrm{~nm}$. Data are reported as percent $590 \mathrm{~nm}$ fluorescence relative to a $0 \mathrm{dpi}$ baseline taken for each $\mathrm{CO}$.

\section{Immunohistochemistry labeling and quantification}

Prior to tissue processing, some COs were treated according to the manufacturer's protocol with a fixable SR-VAD-FMK poly caspase probe (ImmnoChemistry Technologies) to detect activated caspases. At indicated time points, either whole or half COs were collected from culture and placed in 10\% neutral buffered formalin for 1-3 days and protected from light. COs were prepared, cryosectioned, and immunolabeled as previously described [22]. Primary antibodies used were LACV (in- house generated polyclonal mouse, 1:500) and active caspase 3 (Promega\#G7481, 1:250) or Sox2 (abcam \#97959, 1:1,000 or DCX (abcam \#18723, 1:500) or BIII tubulin (abcam \#18207, 1:5000) or NeuN (abcam \#104225, 1: 2000). Secondary conjugated antibodies used goat antimouse 488, donkey anti-rabbit 594 or donkey anti-rabbit 647 (Molecular Probes, 1:500). High-resolution images were taken on a Zeiss Axio Scan.Z1 with the $\times 40$ objective or on a Zeiss 710 LSM with the $\times 63$ objective. For quantification, thresholds for Sox2, DCX, or BIII tubulin positive signal labeling in whole-organoid scans were determined, and the area of positive signal was obtained. This area was then converting into a percent area by dividing by the area of the entire section as determined by staining of cell nuclei and an overlaid brightfield image. Thresholds for positive signal was determined to be $1.25 \times$ the average pixel intensity obtained from secondary only labeled control tissues and above. Images were processed in Imaris v8.4.1 or Fiji.

\section{Single-cell RNA sequencing and analysis}

Mock and infected COs were dissociated as described above with the exception that washes and collections were done in $1 \times$ PBS with $1 \%$ bovine serum albumin. Cells were counted and diluted to $10^{6} / \mathrm{mL}$ prior to loading onto the $10 \times$ Genomics Chromium Controller instrument to be individually partitioned into nanoliter scale droplets within an oil emulsion. RNA sequencing libraries were constructed using the Chromium Single cell 3' Reagent kit V2 (10× Genomics PN-120237) and assayed using a Bioanalyzer 2100 (Agilent) according to the respective manufacturer's instructions. Samples were sequenced using a HiSeq 2500 with a Rapid Paired-End flow cell and cluster kit and Rapid SBS chemistry for a 26/8/98 cycle run (Illumina). Sequencing reads were demultiplexed and mapped to Human Genome build GRCh38.p5 using the Cell Ranger software package (version 2.1.0) from 10× Genomics. Downstream single-cell RNA-Seq analysis was performed using the $\mathrm{R}$ software package Seurat (version 2.3.1) [23]. Briefly, canonical correlation analysis (CCA) was performed on data from the two sample types: mock and LACV COs. CCA subspaces were combined for $t$-distributed stochastic neighbor embedding ( $t$-SNE) analysis, clustering, and visualization.

\section{Flow cytometry}

Mock and infected COs were dissociated as described above and labeled with Fixable Viability Dye eFluor780 (eBioscience, 1:1000) for $30 \mathrm{~min}$ in the dark on ice. Intracellular and nuclear labeling and data collection and analysis were performed as previously described [24]. Antibodies used: Trustain FcX ${ }^{\mathrm{TM}}$ (Biolegend \#422301, 1: 1000), Sox2 (Millipore \#FCMAB112, 1:50), DCX (BD 
Pharmigen \#561505, 1:50), and $\beta I I I$ tubulin (R\&D Systems \#IC1195C, 1:50).

\section{Statistical analysis}

All statistical analyses were carried out in Prism 7.0c and are described in the figure legends.

\section{Results}

iPSC-derived human cerebral organoids are permissive to LACV infection and replication

The consequence and outcomes of LACV infection in human neural tissue are poorly studied. Thus, we infected human-derived iPSC cerebral organoids (COs) with $10^{3}$ or $10^{1} \mathrm{PFU}$ of the virus isolated from post-mortem human brain tissue [25]. Visually, the overall size of infected COs did not change over time (Fig. 1a-c). However, structural density and complexity appeared to be reduced by $6 \mathrm{dpi}$ in the $10^{3}$ PFU (Fig. 1c, f), but not in the $10^{1} \mathrm{PFU}$ (not shown) or mock-infected COs (Fig. 1a, d, white arrows). Structural features were still observed at $3 \mathrm{dpi}$ in COs infected with $10^{3}$ LACV (Fig. 1b, e, white arrows). In histological samples, these areas of structural complexity correlated with nuclei-dense ventricular zone-like regions surrounded by cells undergoing neurogenesis as indicated by Doublecortin (DCX) expression (Fig. 1g). These regions (Fig. 1g, white boxes) became the focus for subsequent histological analysis.

To determine viral replication kinetics in COs, quantitative real-time (qRT) PCR analysis and plaque assays were performed. LACV RNA expression in COs infected with the $10^{1}$ PFU LACV showed weak positive signal in 2 of 3 COs (Fig. 2a), but no detectable infectious virus in media supernatants by plaque assay (Fig. 2b) demonstrating little-to-no replicating virus. In contrast, COs infected with a $10^{3}$ PFU dose showed an $\sim 10$-fold daily increase in LACV RNA expression from 1 to 3 dpi compared to Gapdh reference (Fig. 2a). Relative viral RNA expression remained stable in infected COs from 3 to 6 dpi suggesting viral replication had peaked by 3 dpi. Similarly, viral titers in media supernatants from COs infected with $10^{3}$ PFU LACV peaked and plateaued at $3 \mathrm{dpi}$, remaining stable through 6 dpi (Fig. 2b). These findings demonstrate human iPSC-derived $\mathrm{COs}$ are susceptible to infection by LACV and support viral replication at a $10^{3} \mathrm{PFU}$ dose, causing reduced organoid structural complexity by 6 dpi. Thus, all subsequent infections were performed with $10^{3}$ PFU of LACV.

\section{Cells in LACV-infected iPSC-derived human COs undergo caspase3-dependent cell death}

To examine whether LACV infection of the organoid resulted in neuronal death, we monitored cellular viability of mock- and LACV-infected COs through time using a resazurin reduction-based colorimetric assay. Mock-infected samples converted increasing amounts of resazurin substrate compared to baseline readings throughout experiments (Fig. 3a), suggesting these COs were still growing, although they did not visually appear to increase significantly in size. In contrast, LACV-infected COs converted equivalent or decreasing amounts of resazurin substrate during the experiments compared to baseline (Fig. 3a), with significantly less conversion of resazurin than mockinfected COs by $3 \mathrm{dpi}$. The decreased amount of resazurin substrate conversion in LACV-infected COs indicated these COs had less cellular metabolism which could be a result of cell death.

LACV infection has been shown to induce neuronal cell death through an apoptotic mechanism in both mouse and human neural cell lines $[8,9,26]$. To directly examine apoptosis, we analyzed COs by immunohistochemistry for active caspase- 3 and LACV antigen. Supporting the observed reduction of structural complexity (Fig. 1a-f) by LACV infection, Hoechst nuclear staining showed clear structural features in mock COs (Fig. 3b), but a reduction in these features in LACV-infected COs at 6 dpi (Fig. 3d). Active caspase-3 antigen could be found in a few cells in mock-infected COs at $3 \mathrm{dpi}$ (Fig. 3b), indicating a low level of apoptosis in these organoids in the absence of infection. In contrast, widespread staining for active caspase- 3 was found in LACVinfected organoids at $3 \mathrm{dpi}$ (Fig. 3c) and was even more pronounced at 6 dpi (Fig. 3d). Active caspase- 3 antigen was commonly found in LACV-infected cells (Fig. 3e) but was also found in cells that were negative for LACV suggesting infection can have a negative bystander effect on human neurons (Fig. 3f, white arrows). Conversely, some cells appear to be infected with LACV, but did not express active caspase- 3 antigen suggesting some cells may be resistant to LACV-induced apoptosis (Fig. 3f, yellow arrow).

\section{LACV infection-induced apoptosis occurs more frequently in committed neurons}

To directly examine if there was a difference in which neural developmental stages were being infected and/ or undergoing apoptosis, we labeled mock- and LACV-infected COs at 3 dpi with FLICA SR-VADFMK reagent to detect activated, pro-apoptotic caspases within cells, as well as individual antibodies used to detect virus and neural-lineage cells at distinct development stages (Fig. 4). Sox2 expression was used as a marker of neural progenitor cell, Doublecortin (DCX) for immature committed neurons, and class III $\beta$-tubulin ( $\beta$ III-tubulin) for mature neurons. All three of these labels have been used previously to label respective neuronal populations in human iPSCderived COs [13]. Mock-infected COs contained populations of all three groups of cells with relatively few 


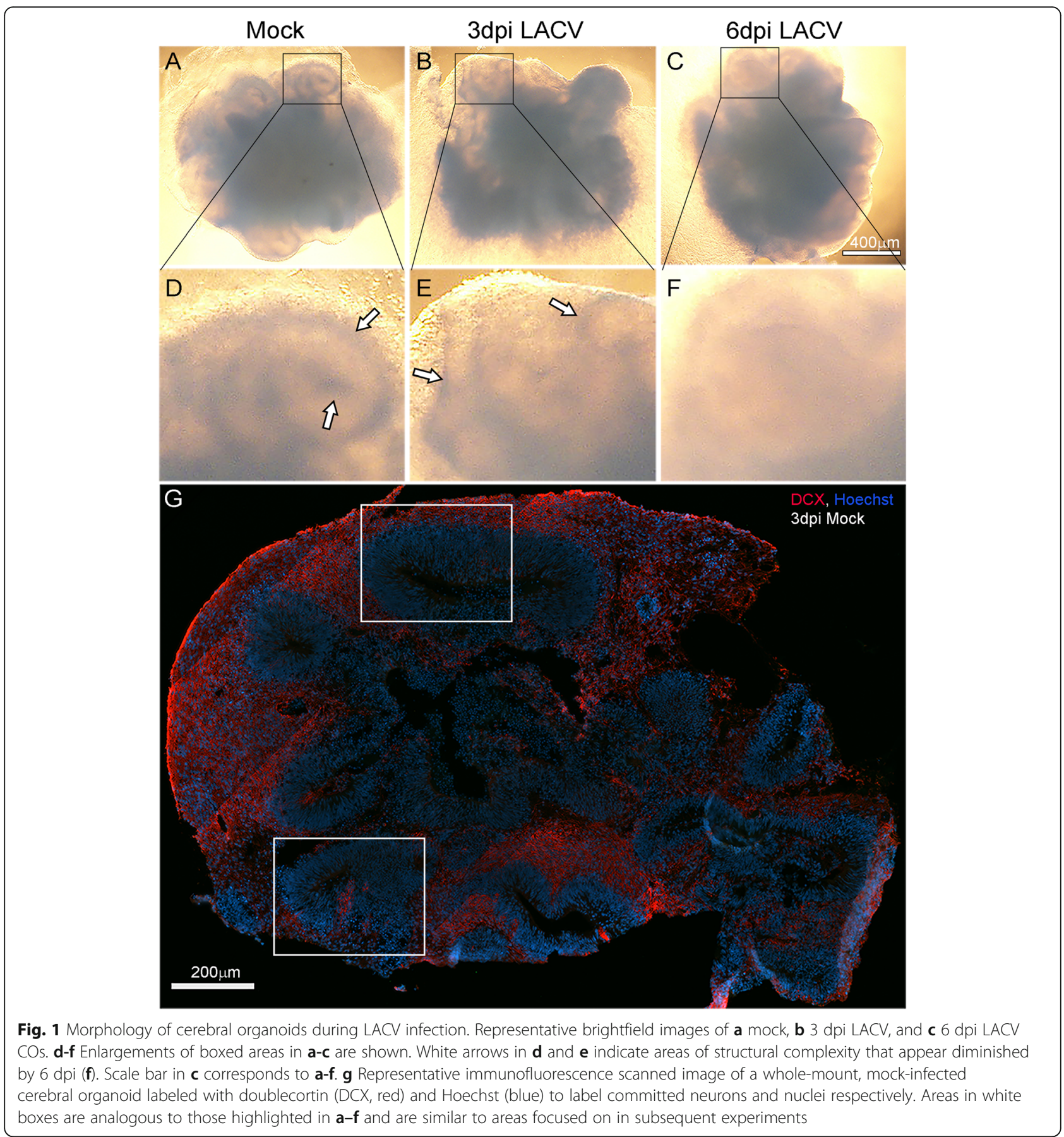

cells staining for active pro-apoptotic caspases (Fig. 4a, $\mathrm{f}, \mathrm{k}$ ). In LACV COs, Sox $2^{+}$progenitor cells (Fig. 4a) were commonly infected (LACV, green), but were rarely positive for active caspases (magenta) at $3 \mathrm{dpi}$ (Fig. $4 \mathrm{~b}-\mathrm{e}$, inset representative of $\mathrm{Sox}^{+}$cell in infected $\mathrm{CO}$ ). In the same organoids, $\mathrm{DCX}^{+}$(Fig. $4 \mathrm{~g}-\mathrm{j}$,) and BIII-tubulin ${ }^{+}$(Fig. 4l-o) cells were commonly associated with areas of dense activated caspase labeling and LACV antigen. Individual $\mathrm{DCX}^{+}$(Fig. 4j, inset) and BIII-tubulin ${ }^{+}$(Fig. 4o, inset) cells were commonly found to be positive for both LACV and active caspases, suggesting these cells were actively undergoing apoptosis.

Cells expressing committed neuron transcriptome profiles are reduced in LACV-infected COs

Single-cell transcriptomic analysis has previously been used to identify neural-lineage cells of differing developmental stages in human cerebral organoids [27]. Therefore, single-cell RNA sequencing was performed on 


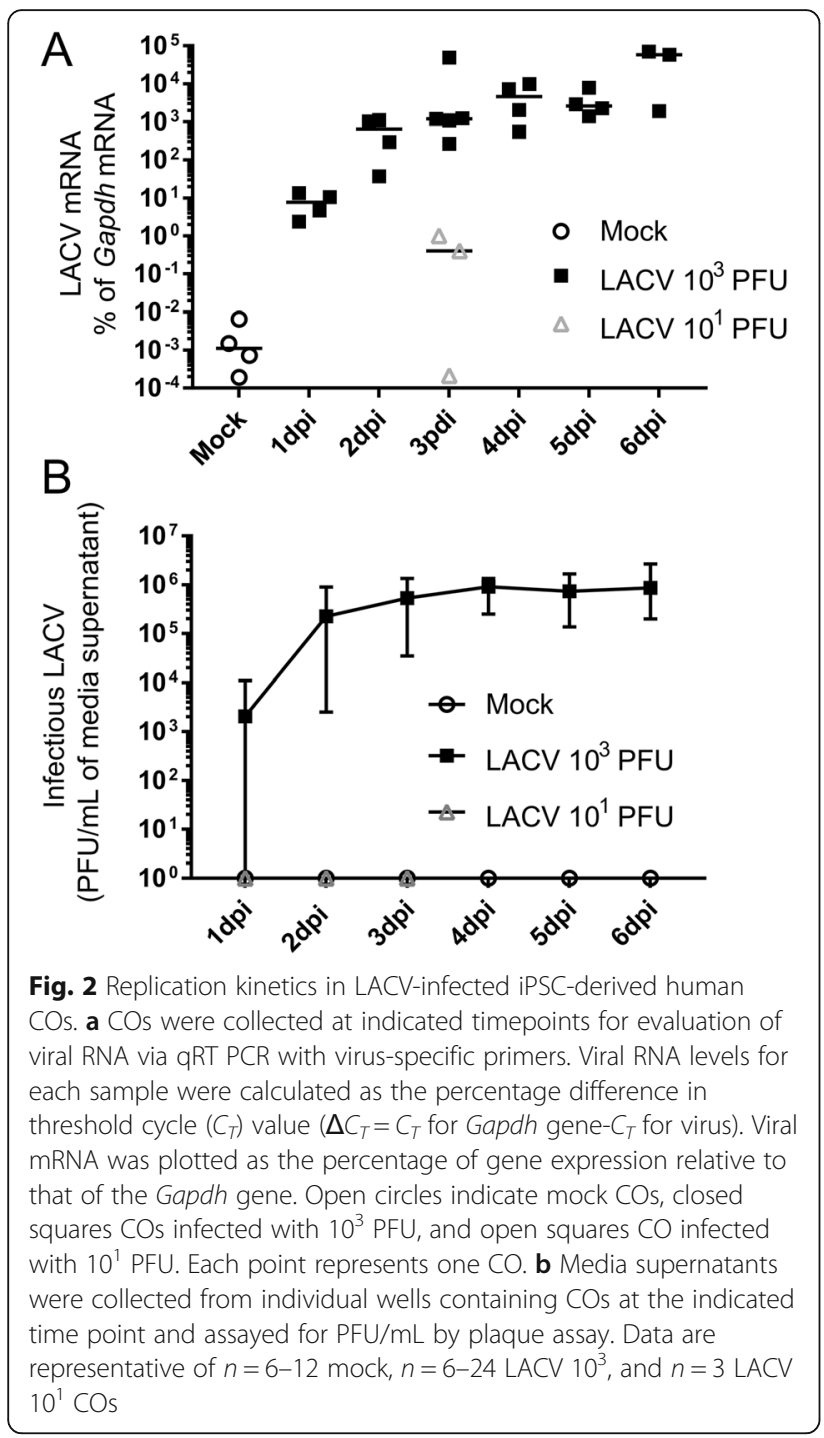

3 dpi mock- and LACV-infected COs. Sequences from 1040 cells from mock and 496 cells from LACV COs were computationally aligned and integrated into 7 clusters (C1-C7) using canonical correlation analysis (CCA) and plotted using t-distributed stochastic neighbor embedding (tSNE) to visualize cellular transcript relatedness in 2D (Fig. 5a). Expression of neural progenitor and committed neuron transcripts was examined within each cluster of this aligned population to determine the predominate cellular development stage (Fig. 5b). Three clusters $(\mathrm{C} 0, \mathrm{C} 3$, and $\mathrm{C} 4)$ had transcriptomic profiles that could not be easily classified and were not consistent with neural progenitor or committed neuron gene profiles. Another cluster, C5, expressed high levels of COL3A1 consistent with extracellular matrix-producing mesenchymal cells that provide structure to COs [27]. Clusters C2, C6, and C7 primarily contained cells expressing transcripts such as SOX2, ASPM, PLK1, PAX6,
RSPO2, INSC, and VIM which have been shown to be associated with neural progenitors [27-33]. Cluster C1 primarily expressed high levels of transcripts such as DCX, TUBB3 (BIII tubulin), SOX11, TAGLN3, MYT1L, and TBR1 and decreasing amounts of VIM which have been associated with cells undergoing neurogenesis and committed neurons [16, 27, 32-34]. Thus, single-cell analysis clearly identified and segregated neural lineage cells of differing developmental stages into these clusters with both neuronal progenitor $(\mathrm{C} 2, \mathrm{C} 6, \mathrm{C} 7)$ and committed neuron $(\mathrm{C} 1)$ populations.

To determine whether LACV infection-induced apoptosis (Figs. 3 and 4) resulted in cellular dropout in specific neural-lineage subpopulations, cells input from mock (Fig. 5c) or LACV (Fig. 5d) COs were identified within the 2D alignment and plotted on two separate tSNE plots, still grouped within their assigned clusters. Cells from individual clusters were then quantified as a percent of input cells from mock or infected COs (Fig. 5e). The percent of input cells in the neural progenitor clusters $(\mathrm{C} 2,6$, and 7$)$ were similar between mock and LACV samples demonstrating equivalent proportions of neural progenitors in both samples. In contrast, the percentage of cells in the committed neuron (C1) cluster was decreased by $41 \%$ in the infected sample compared to the mock (Fig. 5c, d, ellipses and e, black arrow). Thus, LACV infection of COs reduced the number of cells within $\mathrm{C} 1$ but not within the clusters corresponding to cells expressing neural progenitor transcripts.

\section{Quantitative analysis of LACV infection-induced caspase activity within neural-lineage subpopulations of human $\mathrm{COs}$}

To directly quantify the level of apoptosis in different neuronal populations following LACV infection, cells from mock and infected COs were isolated and analyzed using flow cytometry. COs labeled with FLICA SR-VAD-FMK reagent to detect apoptotic cells were non-enzymatically dissociated and labeled with a live-dead stain and antibodies to detect virus and neural-lineage phenotypes. Gating on live cells of specific neural-lineage phenotypes demonstrated that mock samples (Fig. 6a-d) contained some apoptotic cells (Fig. 6a, top left quadrant) as had been observed immunohistologically (Fig. 3b), but no LACV positive (Fig. 6a, lower right quadrant) or active caspase $^{+} /$ $\mathrm{LACV}^{+}$double-positive cells (Fig. 6a, top right quadrant). In contrast, infected COs (Fig. 6e-h) had increased proportions of all three of these groups relative to mock (Fig. 6e). Gating on Sox $2^{+}$neural progenitor cells (Fig. $6 \mathrm{~b}, \mathrm{f}$ ), $\mathrm{DCX}^{+}$ immature neurons (Fig. 6c, g), and $\beta$ III-tubulin ${ }^{+}$mature neurons (Fig. 6d, h) in mock or infected COs allowed for comparison of caspase activation and LACV infection within these neural-lineage subpopulations. Plotting of individual COs showed similar proportions of LACV-infected 

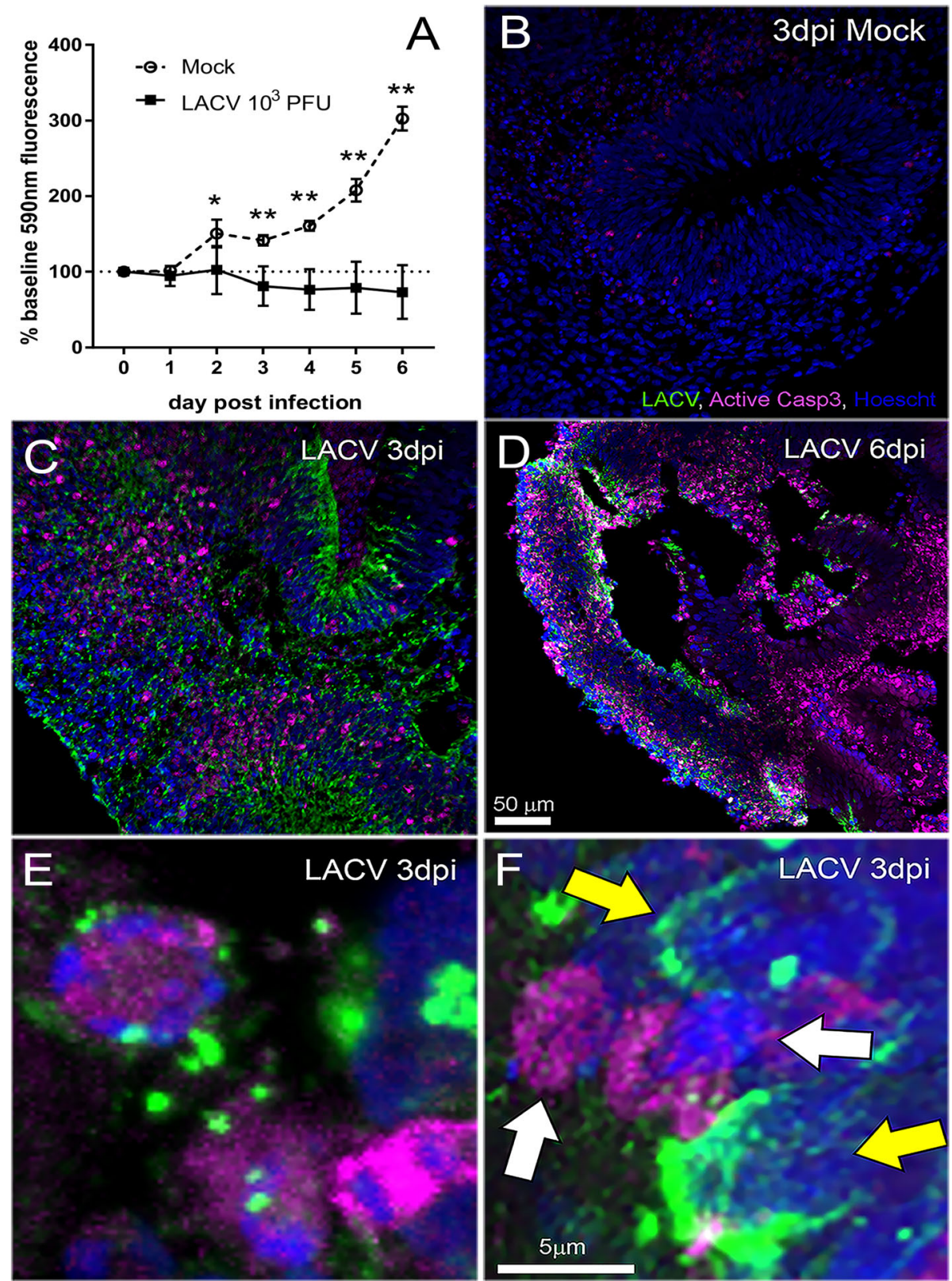

Fig. 3 Cell death in LACV-infected iPSC-derived human COs. a A resazurin reduction-based cell viability assay was performed daily on individual COs within a 24 well plate. Data is plotted as an average percent of base fluorescence measured at $590 \mathrm{~nm}$ for each CO just prior to infection with mock (open circles) or LACV (closed squares). Fluorescence for each sample at each time point was read in triplicate at the indicated time point. A two-way ANOVA with a Sidak's multiple comparisons test was used to determine significance. ${ }^{*} p<0.05,{ }^{* *} p<0.001$. Data are representative of $n=3$ mock and $n=6 \mathrm{LACV} 10^{3} \mathrm{CO}$. $\mathbf{b}$ - $\mathbf{d}$ Representative images of COs immunohistochemically labeled for active caspase-3 (Casp3, magenta), LACV (green), and nuclei (blue). b is from a mock-infected CO, c a 3 dpi LACV-infected organoid and d a 6 dpi LACV-infected organoid. Scale bar in $\mathbf{d}$ also applies to $\mathbf{b}$ and $\mathbf{c}$. e, $\mathbf{f}$ High magnification images taken from the same section of a 3 dpi LACV-infected CO. e Illustrates a caspase $3^{+}$cell (magenta) that is infected with LACV (green). f Caspase $3^{+}$cells that are not infected with LACV (white arrows) and LACV-infected cells that are not caspase $3^{+}$(yellow arrows). Scale bar in $\mathbf{f}$ also applies to $\mathbf{e}$

cells between each subpopulation in infected COs (Fig. 6i). However, proportionally more committed neurons $\left(\mathrm{DCX}^{+}\right.$ or $\beta$ III tubulin ${ }^{+}$) were positive for active caspase than neural progenitors $\left(\mathrm{Sox}^{+}\right)$in infected COs (Fig. 6j). Additionally, the proportions of active caspase positive committed neurons in infected COs were increased relative to mock controls demonstrating virus-specific apoptosis of these neuronal populations (Fig. 6j). Gating on $\mathrm{LACV}^{+}$cells also showed more apoptosis in committed neurons compared to neural progenitors, particularly within the $\mathrm{DCX}^{+}$ 


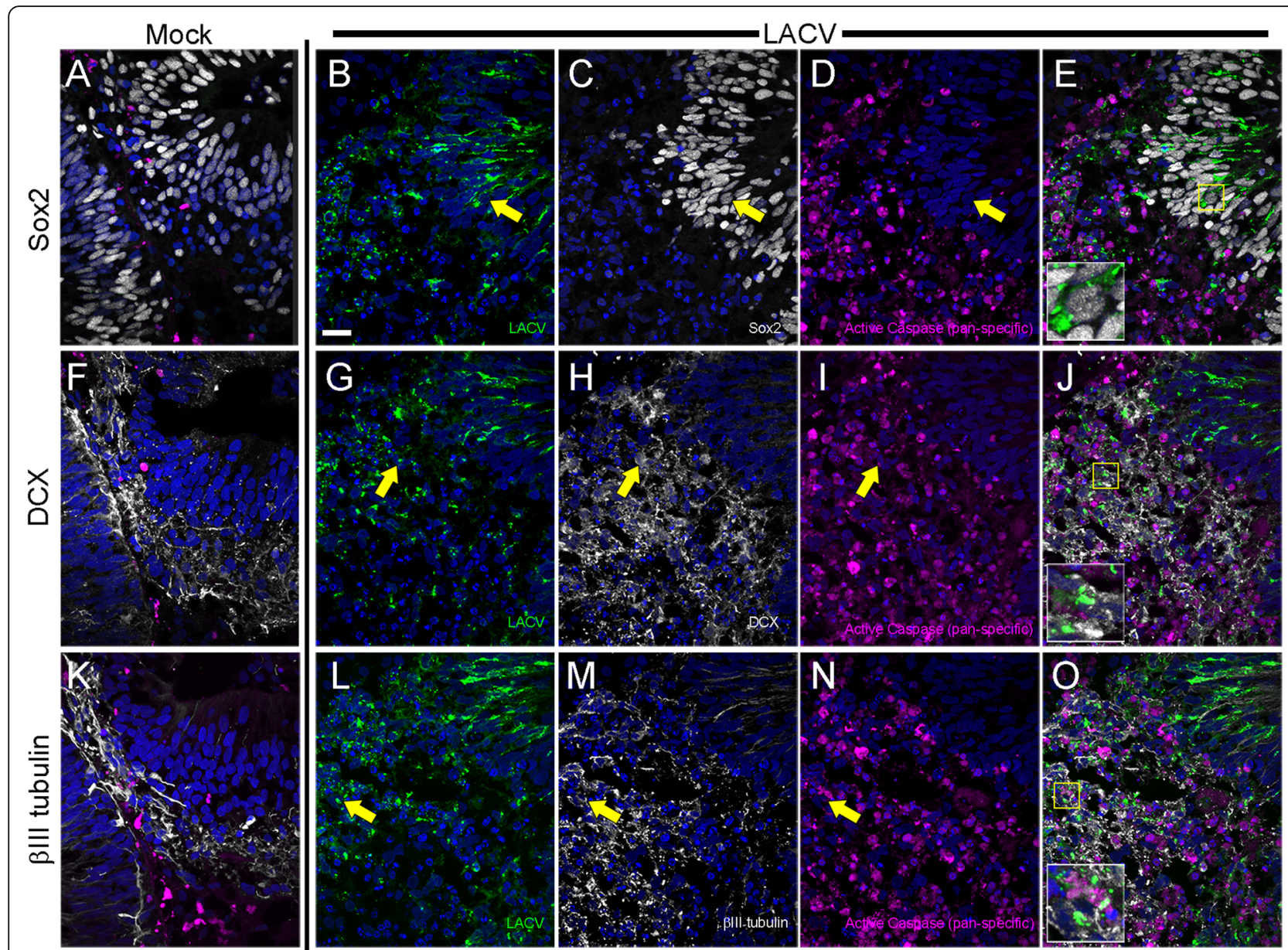

Fig. 4 Committed neurons are susceptible to LACV-induced apoptosis. Representative confocal images of (a, $\mathbf{f}$, and $\mathbf{k})$ mock or (b-e, $\mathbf{g}-\mathbf{j}$, and $\mathbf{I}$ o) 3 dpi LACV-infected COs immunohistochemically labeled with LACV (green), neuronal phenotyping antibodies (white), activated poly caspases (magenta), and nuclei (blue). Images are grouped by row using the neuronal phenotyping antibody with Sox2 being top, DCX middle, and $\beta$ III tubulin bottom. The three middle columns are images of single channels overlaid on nuclei from $3 \mathrm{dpi}$ LACV-infected COs that are labeled accordingly. The far-right column (e, j, and $\mathbf{o})$ is a combination of all four labels. The insets in $\mathbf{e}, \mathbf{j}$, and $\mathbf{o}$ are enlarged images of the highlighted yellow boxes in each panel. The corresponding yellow arrows in the associated individual label panels highlight the cell of interest shown in the inset. The images in the $\mathbf{a}, \mathbf{f}$, and $\mathbf{k}$ mock column are a combination of all four labels. All images were taken with a $\times 63$ objective and are maximum intensity projects of $3 \mu \mathrm{m} z$-stacks taken with a $0.5 \mu \mathrm{m}$ step. Scale bar in $B=20 \mu \mathrm{m}$ and applies to all panels

population (Fig. 6k). Further complementing this finding, the overall number of $\mathrm{DCX}^{+}$and $\beta$ III-tubulin ${ }^{+}$committed neurons was reduced in LACV-infected COs compared to mock COs (Fig. 6g, h vs. c, d, bottom left quadrants). In contrast, the number of neural progenitors was relatively similar between mock and infected (Fig. $6 \mathrm{f}$ vs b, bottom left quadrant). Together, these data suggest that committed neurons are more susceptible to LACV-induced apoptosis than neural progenitors.

The type I interferon response to LACV is more robust in neural progenitors than committed neurons

Further examination of genes from each cluster in our single-cell sequencing experiment of $3 \mathrm{dpi}$ mock vs LACV-infected COs identified several interferon (IFN)-stimulated genes (ISGs) as differentially expressed between committed neurons (C1) and neural progenitors (C2, C6, C7) (Fig. 7, upper section). Neural progenitor clusters from the LACV CO all contained proportionally greater numbers of cells expressing higher amounts of ISG transcripts relative to mock demonstrating a robust IFN response. By comparison, the cluster containing committed neurons $(\mathrm{C} 1)$ had a much weaker and less inclusive ISG response suggesting that even the surviving committed neurons had a limited IFN response. To further validate these findings, transcripts of the IFN signaling pathway were also compared between the mock and LACV COs (Fig. 7 lower section). Levels of IFN receptor and downstream signaling effector transcripts were proportionally increased in neural progenitor clusters in the LACV-infected organoid 


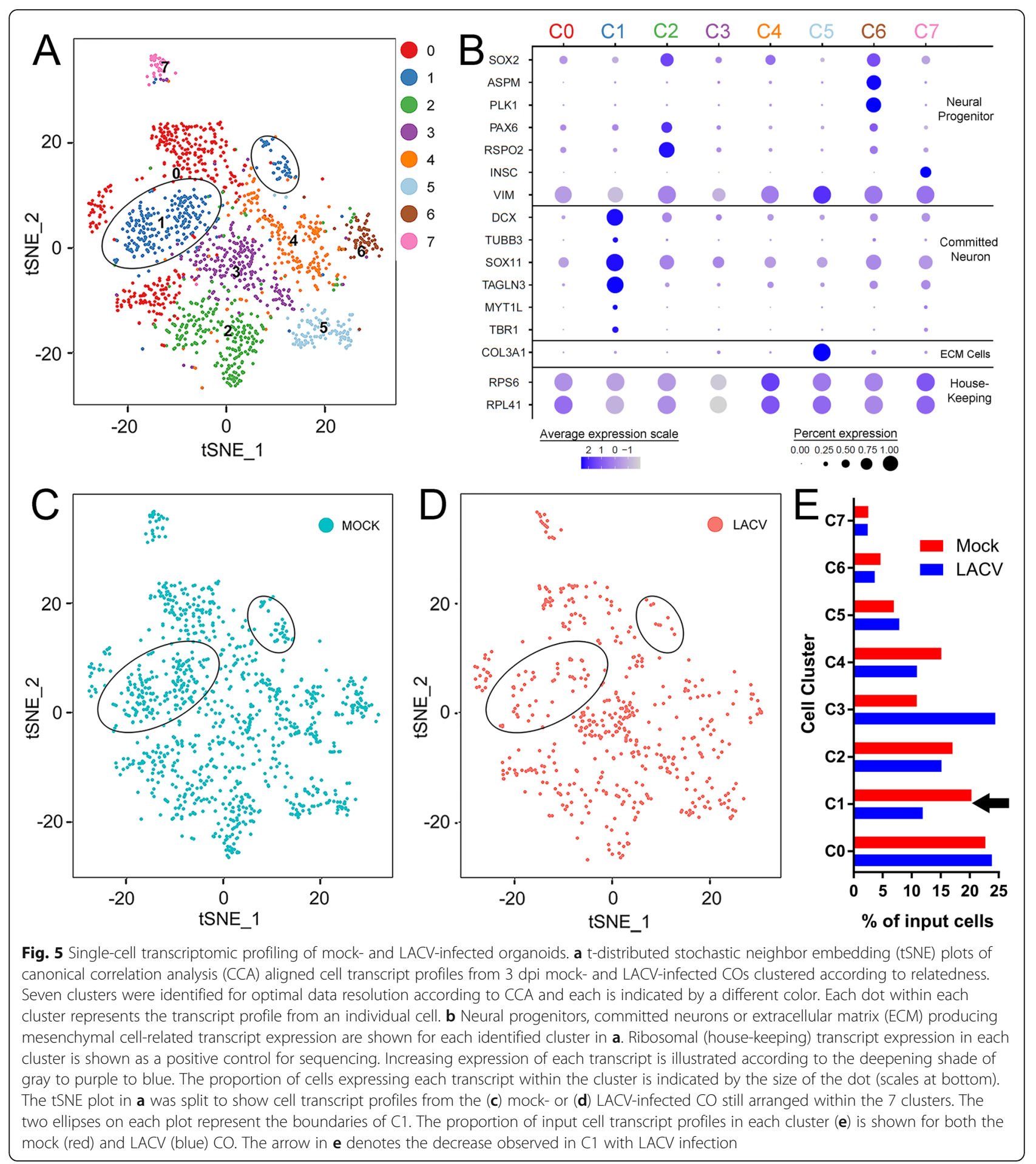

relative to mock. In contrast, the proportions of these transcripts were largely unchanged in the committed neuron cluster when mock was compared to LACV infection suggesting signaling was impaired in the cells. Thus, neural progenitors in the infected organoid mounted a robust IFN signaling and ISG response that was not found in committed neurons.

\section{Exogenous administration of type I IFN inhibits LACV- induced cell death in $\mathrm{CO}$}

Due to the differential IFN signaling and ISG responses of neural progenitors and committed neurons in LACVinfected COs (Fig. 7b), we exogenously administered recombinant IFN to LACV-infected organoids $24 \mathrm{~h}$ postinfection to determine if type I IFN activation affected 


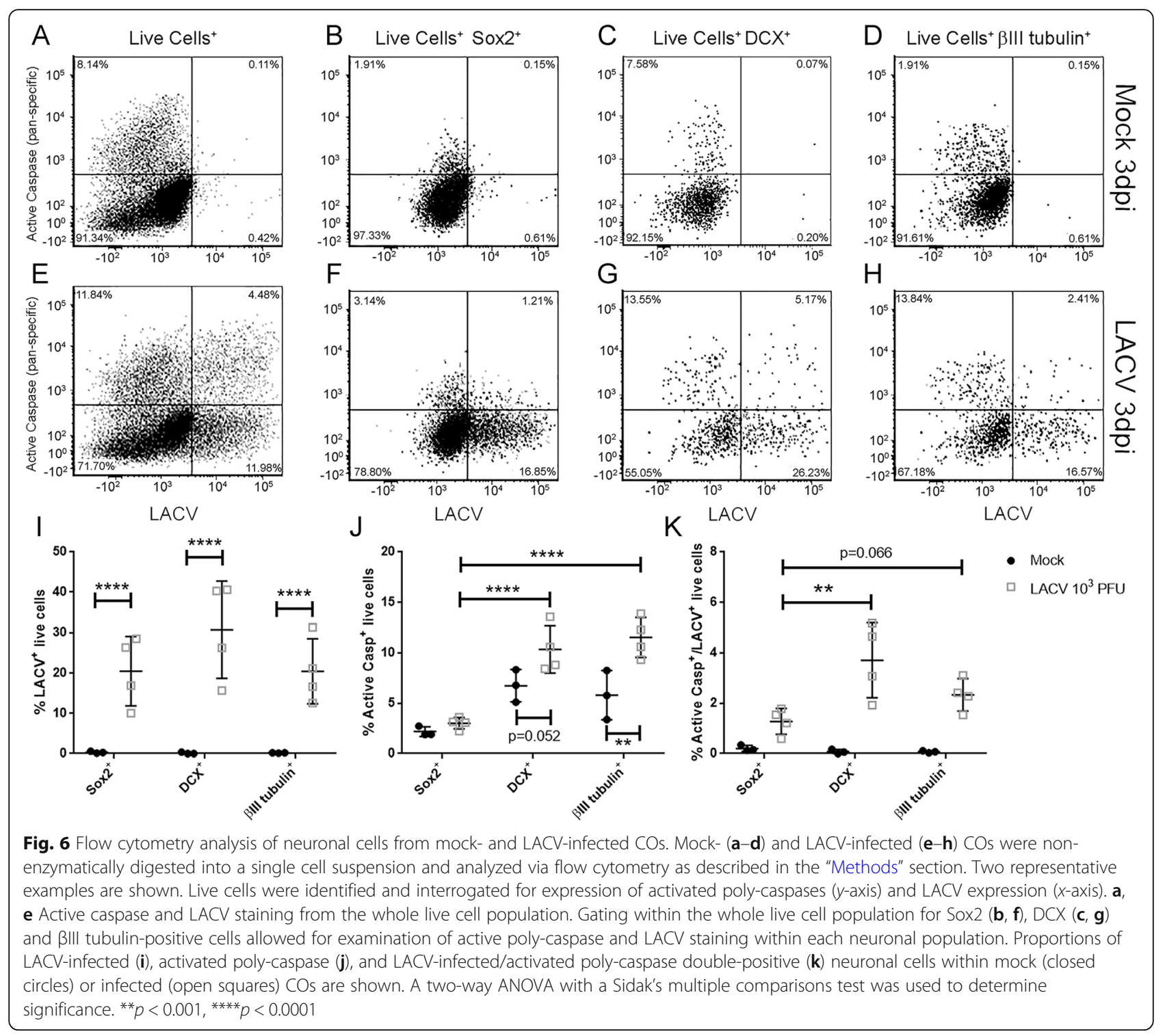

$\mathrm{CO}$ viability. As both $\alpha$ and $\beta$ IFNs have been shown to induce ISG signaling in neural-lineage cells [35], COs were treated with either IFN $\alpha 2$ and IFN $\alpha 4$ concomitantly (Fig. $8 \mathrm{a}$ ) or IFN $\beta 1$ (Fig. 8 b). Both IFN $2 / 4$ and IFN $\beta 1$ treatment of LACV-infected COs resulted in a significant increase in viability compared to vehicletreated infected COs (Fig. 8a, b). However, only IFN $\beta 1$ treatment resulted in sustained increased viability throughout the experiment (Fig. 8b). qRT PCR analysis of supernatants from treated COs showed a modest, but significant, $0.5-1 \log$ decrease in LACV RNA at multiple days during the experiment in IFN-treated COs (Fig. 8c). This finding demonstrated that exogenous application of type I IFN induced activation of antiviral signaling during LACV infection.

To determine a cell-type-specific effect of IFN signaling induction, we immunohistochemically labeled sections from mock IFN $\beta$, LACV vehicle, and LACV IFN $\beta$-treated COs with markers of neural progenitors (Sox2), immature (DCX), and mature ( $\beta$ III tubulin) committed neurons and quantified positive signal as a percent of organoid area (Fig. 8d). On average, neural progenitor Sox2-positive signal was unchanged regardless of infection or recombinant IFN treatment. In contrast, DCX and BIII tubulin expression decreased in the LACV vehicle treatment group relative to the mock IFN $\beta$-treated group, with $\beta$ III tubulin being statistically less (Fig. 8d). This further confirmed our previous findings of committed neuron loss with LACV infection in COs (Figs. 5 and 6). However, DCX and $\beta I I I$ tubulin signal was on average higher in LACV IFN $\beta$-treated COs compared to LACV vehicle. Collectively, these data suggest IFN treatment of LACV-infected COs promotes cell survival primarily 


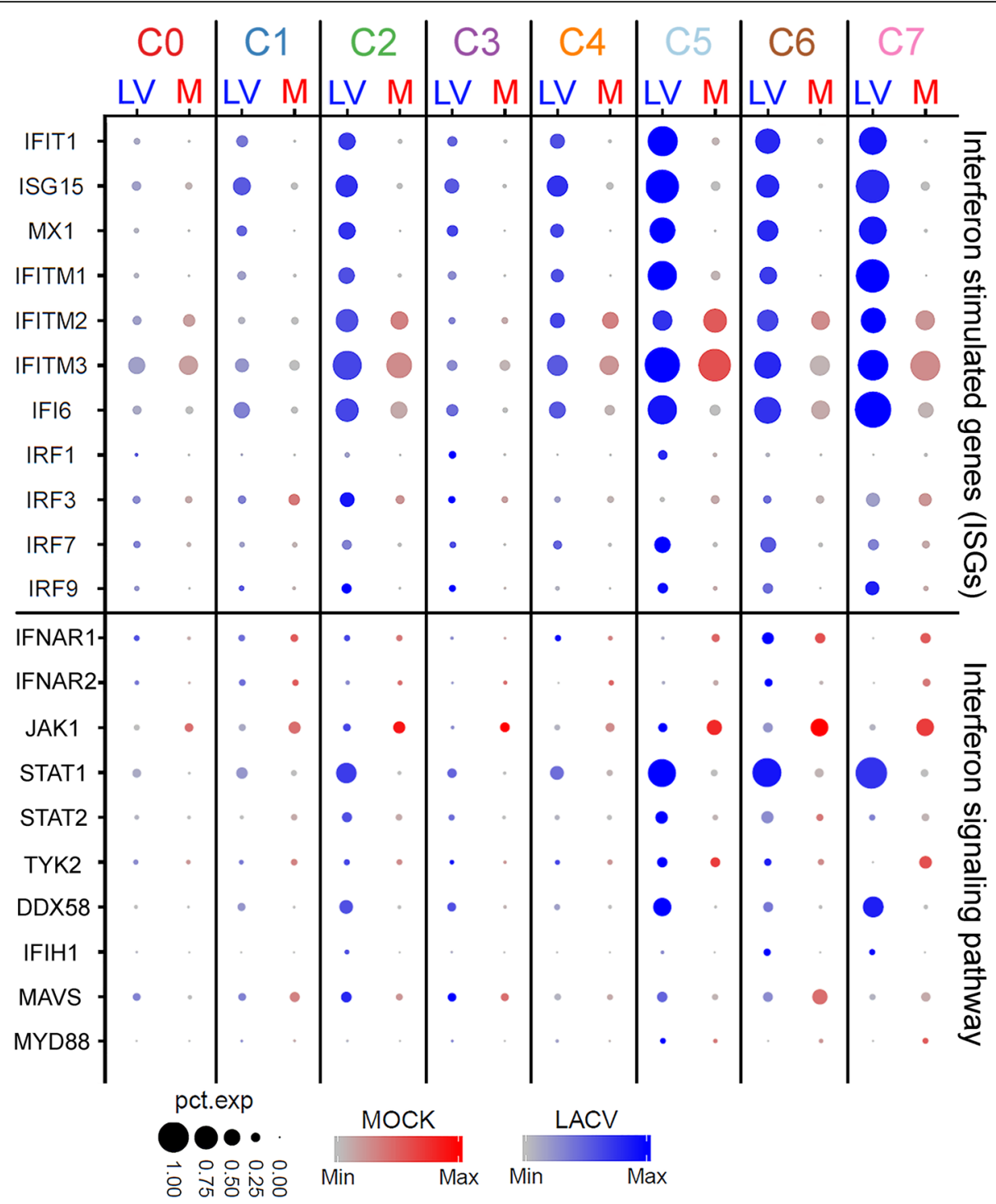

Fig. 7 The type I interferon (IFN) response to LACV in neural progenitors and committed neurons. Expression of interferon-stimulated gene (ISG, upper section) type I IFN signaling and effector transcripts in the mock and LACV cells from COs that underwent single-cell transcriptome analysis. The same 7 clusters that were identified in Fig. 5 are shown and are segregated into the mock (red) and LACV (blue) samples. Increasing expression of each transcript is illustrated according to the deepening shade of either red for mock or blue for LACV. The proportion of cells expressing each transcript within the cluster are indicated by the size of the dot (scales at the bottom)

of committed neurons likely through the activation of the type I IFN signaling and the ISG response.

\section{Discussion}

In monolayer culture, differentiated neurons have been shown to be more resistant to LACV infection-induced apoptosis than undifferentiated neurons [8]. However, this critical question has not been addressed in a complex neural tissue with neurons at various developmental stages. Here, we examined LACV infection in iPSCderived human COs undergoing neurogenesis. We found LACV infection decreased cell viability and increased apoptosis in COs as early as 3 dpi (Fig. 3). By 6 dpi, structural complexity was reduced (Figs. 1f, and 3d) and cell viability decreased relative to mock (Fig. 3a) suggesting the loss of cells undergoing neural development was sustained during active LACV infection. Apoptosis was observed primarily in developmentally committed neurons and less frequently in neural progenitor cells (Figs. 3, 4, and 5) indicating that maturing neurons were more susceptible to virus-induced death.

COs are a recently developed and powerful model with which to study basic neurodevelopment and human neurological disease. This model utilizes human iPSCs treated with specific growth factors and conditions to stimulate the self-assembly of an organized tissue that 


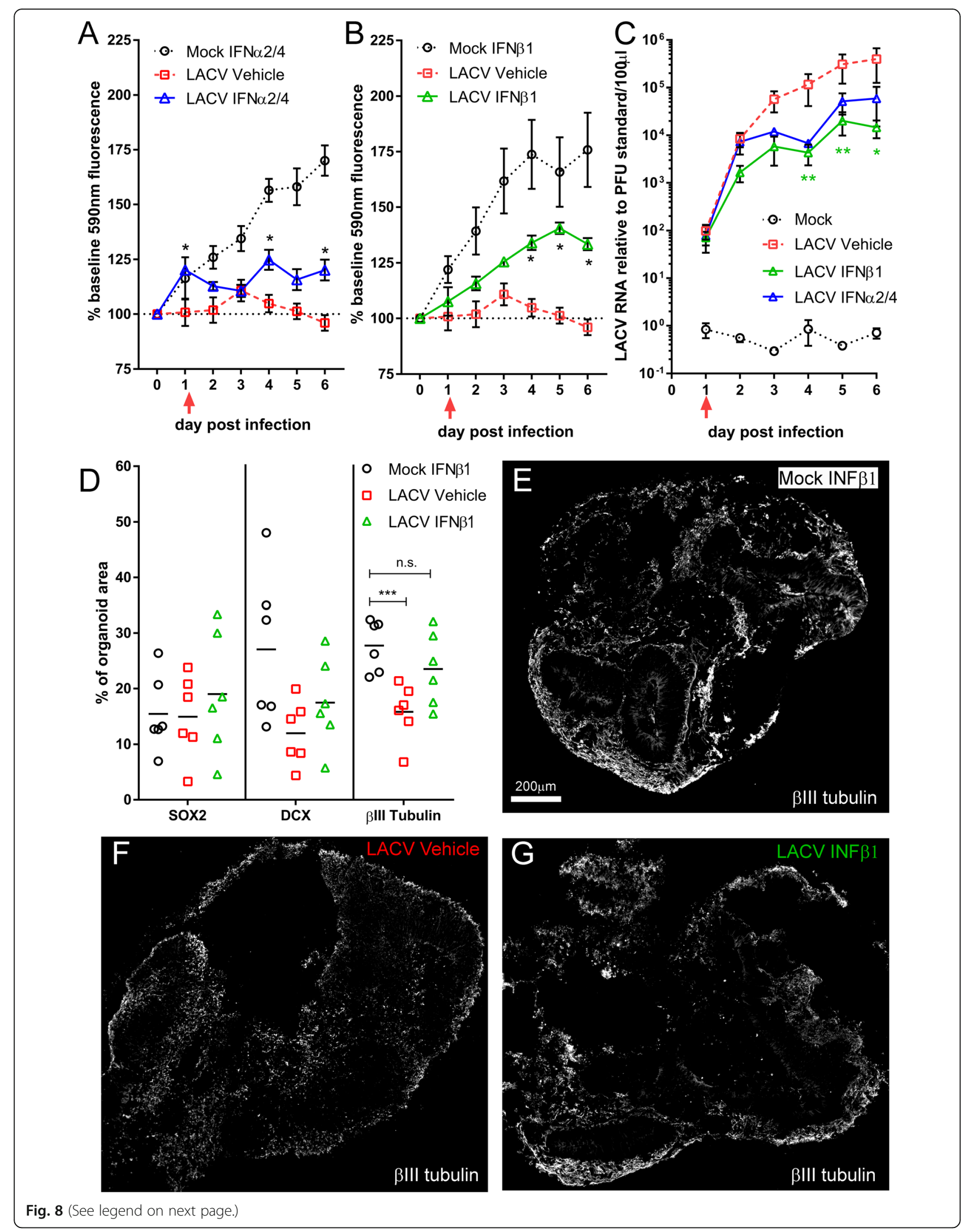




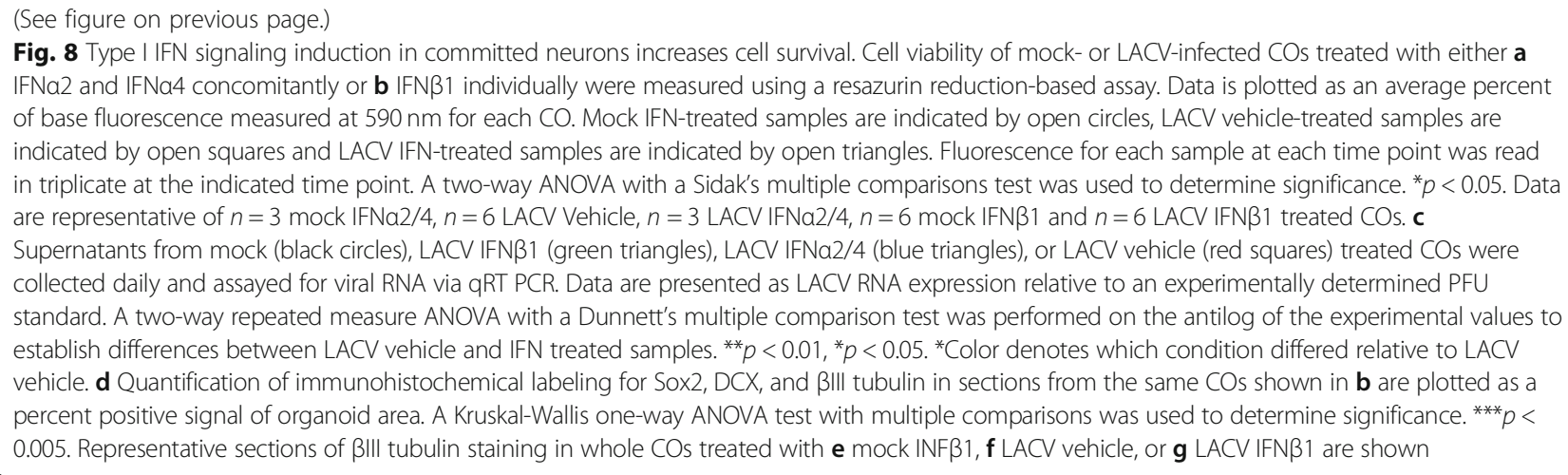

contains most cell types found in the developing brain including neural progenitors, committed neurons, micro-, astro-, and oligodendroglia [13, 18, 36-38]. Previous studies have shown that neurons within COs mature and form functional connections which strengthen through time $[18,39]$. While it remains undetermined when the earliest of these connections form, significant electrical activity is detected in 2-month-old organoids with spiking activity detected at 6 weeks [40]. Similar with these previous studies, we found significant numbers of committed, mature neurons in COs at 21 days of neural induction (Figs. 4, 5, and 6), suggesting neuronal networks could be forming. This time point is prior to the proliferation of glial cells within the $\mathrm{CO}$ which occurs primarily between 30 and 80 days post neural induction [18, 36, 37]. This allowed us to take advantage of this system to specifically examine the response of neuronal cells, from progenitors to mature cells, to LACV infection with minimal confounding influence of glial cells.

Our study contrasts with previous work where differentiated cell were more resistance to LACV-induced apoptosis [8]. However, the cells in this previous study were derived directly from a pluripotent human testicular embryonal carcinoma and induced toward a neural ectodermal lineage [41]. As such, those cells do not undergo a typical developmental neurogenic program and may not express key determinates of susceptibility that would be present in cells of the developing human brain. In contrast, COs undergo a neurogenic program similar to normal human cortical development [13] and contain heterogenous neural-lineage cell types, such as radial glial cells, that provide important trophic and developmental cues [42] that are lacking in homogenous neural cultures [43]. Thus, COs provide a more relevant model of LACV infection in a developing human brain, suggesting that the finding that committed neurons are more susceptible to virus-induced apoptosis may be more accurate than findings from monocultures.

Our single-cell sequencing analysis demonstrated that cells with the transcriptional profile of committed neurons were decreased by LACV infection. However, another undefined population (C3) increased with infection (Fig. 5e). This population did not express transcripts consistent with neural progenitors or committed neurons. However, like the committed neurons cluster (C1), VIM expression was minimal (Fig. 5b). VIM encodes the intermediate filament vimentin which is typically expressed by neural progenitors, but its expression decreases as these progenitor cells mature and become committed neurons [33]. Thus, this cluster may contain cells that have undergone abnormal transcriptional maturation as a consequence of viral infection, as has been demonstrated with other pathogens such as cytomegalovirus [44]. These cells may be derived from another undefined cluster (C4) that has some SOX2 and VIM expression (Fig. 5b) and also shows a modest proportional decrease in cell number with infection (Fig. 5e). Further analysis of these cells at multiple time points post-infection could provide evidence on how LACV infection alters the transcriptional profile of both neural progenitors and committed neurons.

Our flow cytometry data demonstrate an increase in the proportion of $\mathrm{LACV}^{+}$apoptotic immature and mature neurons in infected COs indicating a cytotoxic effect of virus infection (Fig. 6). However, our histological data (Figs. 3f and 4) and flow analysis (Fig. 6) indicate that not all apoptotic cells are infected suggesting that some cells are dying due to a secondary effect of viral infection. This phenomenon occurs in other in vivo models of viral encephalitis [45-47] and has primarily been attributed to glial and immune activation [48-50]. However, because glial immune responses are largely absent from COs at this developmental timepoint $[13,36]$, this implies the cytotoxic trigger(s) originate from neural-lineage cells. Developing neurons within COs establish functional neuronal networks $[18,39]$, the connectivity of which is required to prevent activation of intrinsic apoptotic cellular programs [51]. Thus, uninfected maturing neurons may die as a result of losing connectivity with other infected apoptotic neurons. 
Alternatively, these neurons may undergo apoptosis following bystander activation of their innate immune responses. Previous studies by our lab and others have shown that neurons undergo apoptosis following tolllike receptor (TLR) signaling through endocytosed viral RNA or micro-RNA produced by dying neurons [52-54]. The environment of infected, dying neurons in the organoids may provide sufficient stimuli to induce TLR-mediated neuronal apoptosis.

While well defined in immune cells, the type I IFN response to virus infection in human neural-lineage cells has not been well characterized. We found that $\mathrm{CO}$ neural progenitors, but not committed neurons have a robust IFN signaling and ISG response to LACV (Fig. 7) correlating with resistance to virus-induced apoptosis (Figs. 4, 5, and 6). These findings contrast with two other studies of alphavirus infection of differentiated neurons in monolayers, where enhanced type I IFN responses in differentiated neural-lineage cells correlated with increased resistance to virus-induced cytopathy primarily in differentiated neurons $[35,55]$. Our data clearly indicate that transcripts for IFN signaling and ISGs are primarily induced in neural progenitor cells compared to committed neurons in response to LACV infection (Fig. 7). Although the difference in these studies could be due to the systems used, i.e., monolayers versus organoids, it is also possible this differing response may be due to specific actions of the virus. For example, LACV has been shown to inhibit IFN production by neurons in an in vivo mouse model through actions of its non-structural protein NSs [56]. Possibly, LACV may suppress innate immune IFN signaling in committed neurons more than in neural progenitor cells leading to enhanced apoptosis in this population.

Our data also demonstrate that exogenous administration of recombinant IFN to LACV-infected COs rescues viability (Fig. $8 \mathrm{a}, \mathrm{b}$ ). Because most of the cell death associated with LACV infection was in committed neurons, we reasoned the increase in viability is most likely due to enhanced survival of this cell type. We confirmed this by immunohistochemical quantification of whole sections of treated and untreated LACV- and mock-infected COs (Fig. 8c). Previous studies using IFNs as a treatment in the CNS have shown some negative consequences including inhibition of neurogenesis and promotion of apoptosis when administered at high concentrations [57]. However, IFN $\beta 1$ can also protect cells in the brain from apoptosis in the absence of growth factors [58]. Our current data indicates that induction of IFN signaling and the ISG in committed neurons correlates with increases cell survival during LACV infection and should be further examined as a potential therapeutic for the treatment of viral encephalitis.

\section{Conclusions}

Determining the effect of virus infection in the brain on neuronal subpopulations is critical to our understanding of encephalitic disease. Here, we demonstrate that cerebral organoids are a useful model to study these effects as they contain neurons at multiple developmental stages patterned similarly to a human brain. Experimentally, we show that committed neurons are more susceptible to LACV-induced apoptosis than neural progenitors despite similar levels of infection. This susceptibilty can be explained, at least in part, by the poor type I IFN response we observed in committed neurons compared to progenitors during virus infection. Furthermore, we demonstrate that $\mathrm{CO}$ cell viability can be rescued by induction of type I IFN signaling using recombinant IFNs. Together these findings demonstrate that induction of IFN signaling is a critical determinant in encephalitic virus-induced neuronal death.

\section{Abbreviations \\ CCA: Canonical correlation analysis; CO: Cerebral organoid: \\ DCX: Doublecortin; EB: Embryoid body; IFN: Interferon; iPSC: Induced pluripotent stem cell; ISG: Interferon stimulated gene; LACV: La Crosse virus; PFU: Plaque forming unit; qRT: Quantitative reverse transcription; TLR: Toll- like receptor; tSNE: t-distribution stochastic neighbor embedding; $\beta$ III tubulin: Class III $\beta$-tubulin}

\section{Acknowledgements}

The authors thank Suzette Priola, Audrey Chong, Simote Foliaki, and James Striebel for review of the manuscript and Juraj Kabat for assistance with imaging and image analysis.

\section{Authors' contributions}

CWW, CLH, and KEP conceived of and designed experiments. CWW, TAW, $A B C$, EES CAM, BRG, and SMB performed experiments and provided technical expertise. CWW, CLH and KEP wrote the manuscript. All authors read and approved the final manuscript.

\section{Funding}

This work was supported by the National Institute of Allergy and Infectious Diseases, Division of Intramural Research.

\section{Availability of data and materials}

The sequencing dataset generated and analyzed during the current study will be made publicly available through NCBI GEO (accession\#GSE131434) upon publication. Currently, the dataset can be released through NCBI GEO on a reasonable request to the corresponding author.

\section{Ethics approval and consent to participate}

The human samples used in this study were obtained from a commercial source (ATCC) and the NIH Office of Human Subjects Research Protections (OHSRP) has determined these samples to be exempt from IRB review.

\section{Consent for publication}

Not applicable.

\section{Competing interests}

The authors declare that they have no competing interests.

\section{Author details}

'Laboratory of Persistent Viral Diseases, Rocky Mountain Laboratories, National Institute of Allergy and Infectious Diseases, National Institutes of Health, 903 S. 4th Street, Hamilton, MT 59840, USA. ${ }^{2}$ Research Technologies Branch, Rocky Mountain Laboratories, National Institute of Allergy and Infectious Diseases, National Institutes of Health, Hamilton, MT 59840, USA. ${ }^{3}$ Laboratory of Virology, Rocky Mountain Laboratories, National Institute of 
Allergy and Infectious Diseases, National Institutes of Health, Hamilton, MT 59840, USA.

\section{Received: 9 July 2019 Accepted: 9 October 2019} Published online: 18 November 2019

\section{References}

1. Haddow AD, Bixler D, Odoi A. The spatial epidemiology and clinical features of reported cases of La Crosse virus infection in West Virginia from 2003 to 2007. BMC Infect Dis. 2011;11:29.

2. Thompson WH, Kalfayan B, Anslow RO. Isolation of California encephalitis group virus from a fatal human illness. Am J Epidemiol. 1965;81:245-53.

3. La Crosse Encephalitis Epidemiology \& Geographic Distribution. [https:// www.cdc.gov/lac/tech/epi.html]. Accessed 1 July 2019.

4. McJunkin JE, Khan R, de los Reyes EC, Parsons DL, Minnich LL, Ashley RG, Tsai TF. Treatment of severe La Crosse encephalitis with intravenous ribavirin following diagnosis by brain biopsy. Pediatrics. 1997;99:261-7.

5. McJunkin JE, de los Reyes EC, Irazuzta JE, Caceres MJ, Khan RR, Minnich LL, Fu KD, Lovett GD, Tsai T, Thompson A. La Crosse encephalitis in children. N Engl J Med. 2001;344:801-7.

6. de los Reyes EC, JE MJ, Glauser TA, Tomsho M, O'Neal J. Periodic lateralized epileptiform discharges in La Crosse encephalitis, a worrisome subgroup: clinical presentation, electroencephalogram (EEG) patterns, and long-term neurologic outcome. J Child Neurol. 2008;23:167-72.

7. Balfour HH Jr, Siem RA, Bauer H, Quie PG. California arbovirus (La Crosse) infections. I. Clinical and laboratory findings in 66 children with meningoencephalitis. Pediatrics. 1973;52:680-91.

8. Pekosz A, Phillips J, Pleasure D, Merry D, Gonzalez-Scarano F. Induction of apoptosis by La Crosse virus infection and role of neuronal differentiation and human bcl-2 expression in its prevention. J Virol. 1996;70:5329-35.

9. Dawes BE, Gao J, Atkins C, Nelson JT, Johnson K, Wu P, Freiberg AN. Human neural stem cell-derived neuron/astrocyte co-cultures respond to La Crosse virus infection with proinflammatory cytokines and chemokines. J Neuroinflammation. 2018;15:315.

10. Sorrells SF, Paredes MF, Cebrian-Silla A, Sandoval K, Qi D, Kelley KW, James D, Mayer S, Chang J, Auguste Kl, et al. Human hippocampal neurogenesis drops sharply in children to undetectable levels in adults. Nature. 2018;555:377-81.

11. Gould E, Beylin A, Tanapat P, Reeves A, Shors TJ. Learning enhances adult neurogenesis in the hippocampal formation. Nat Neurosci. 1999;2:260-5.

12. Leuner B, Mendolia-Loffredo S, Kozorovitskiy Y, Samburg D, Gould E, Shors TJ. Learning enhances the survival of new neurons beyond the time when the hippocampus is required for memory. J Neurosci. 2004;24:7477-81.

13. Lancaster MA, Renner M, Martin CA, Wenzel D, Bicknell LS, Hurles ME, Homfray T, Penninger JM, Jackson AP, Knoblich JA. Cerebral organoids model human brain development and microcephaly. Nature. 2013;501:373-9.

14. Gotz M, Huttner WB. The cell biology of neurogenesis. Nat Rev Mol Cell Biol. 2005;6:777-88

15. Stiles J, Jernigan TL. The basics of brain development. Neuropsychol Rev. 2010;20:327-48.

16. Couillard-Despres S, Winner B, Schaubeck S, Aigner R, Vroemen M, Weidner $\mathrm{N}$, Bogdahn U, Winkler J, Kuhn HG, Aigner L. Doublecortin expression levels in adult brain reflect neurogenesis. Eur J Neurosci. 2005;21:1-14.

17. Lancaster MA, Knoblich JA. Generation of cerebral organoids from human pluripotent stem cells. Nat Protoc. 2014;9:2329-40.

18. Pasca AM, Sloan SA, Clarke LE, Tian Y, Makinson CD, Huber N, Kim CH, Park $J Y$, O'Rourke NA, Nguyen KD, et al. Functional cortical neurons and astrocytes from human pluripotent stem cells in 3D culture. Nat Methods. 2015;12:671-8

19. Evans $A B$, Winkler CW, Peterson KE. Differences in neuropathogenesis of encephalitic California serogroup viruses. Emerg Infect Dis. 2019;25:728-38.

20. Butchi NB, Pourciau S, Du M, Morgan TW, Peterson KE. Analysis of the neuroinflammatory response to TLR7 stimulation in the brain: comparison of multiple TLR7 and/or TLR8 agonists. J Immunol. 2008;180:7604-12.

21. Winkler CW, Woods TA, Rosenke R, Scott DP, Best SM, Peterson KE. Sexual and vertical transmission of Zika virus in anti-interferon receptor-treated Rag1-deficient mice. Sci Rep. 2017;7:7176.

22. Winkler CW, Foster SC, Matsumoto SG, Preston MA, Xing R, Bebo BF, Banine F, Berny-Lang MA, Itakura A, McCarty OJ, Sherman LS. Hyaluronan anchored to activated CD44 on central nervous system vascular endothelial cells promotes lymphocyte extravasation in experimental autoimmune encephalomyelitis. J Biol Chem. 2012;287:33237-51.

23. Butler A, Hoffman P, Smibert P, Papalexi E, Satija R. Integrating single-cell transcriptomic data across different conditions, technologies, and species. Nat Biotechnol. 2018;36:411-20.

24. Winkler CW, Myers LM, Woods TA, Carmody AB, Taylor KG, Peterson KE. Lymphocytes have a role in protection, but not in pathogenesis, during La Crosse virus infection in mice. J Neuroinflammation. 2017;14:62.

25. Huang C, Thompson WH, Campbell WP. Comparison of the M RNA genome segments of two human isolates of La Crosse virus. Virus Res. 1995:36:177-85.

26. Mukherjee $P$, Woods TA, Moore RA, Peterson KE. Activation of the innate signaling molecule MAVS by bunyavirus infection upregulates the adaptor protein SARM1, leading to neuronal death. Immunity. 2013;38:705-16.

27. Camp JG, Badsha F, Florio M, Kanton S, Gerber T, Wilsch-Brauninger M, Lewitus E, Sykes A, Hevers W, Lancaster M, et al. Human cerebral organoids recapitulate gene expression programs of fetal neocortex development. Proc Natl Acad Sci U S A. 2015;112:15672-7.

28. Zhang S, Cui W. Sox2, a key factor in the regulation of pluripotency and neural differentiation. World J Stem Cells. 2014;6:305-11.

29. Sakai D, Dixon J, Dixon MJ, Trainor PA. Mammalian neurogenesis requires treacle-Plk1 for precise control of spindle orientation, mitotic progression, and maintenance of neural progenitor cells. PLoS Genet. 2012;8:e1002566.

30. Postiglione MP, Juschke C, Xie Y, Haas GA, Charalambous C, Knoblich JA. Mouse inscuteable induces apical-basal spindle orientation to facilitate intermediate progenitor generation in the developing neocortex. Neuron. 2011;72:269-84.

31. Gyllborg D, Ahmed M, Toledo EM, Theofilopoulos S, Yang S, FfrenchConstant C, Arenas E. The matricellular protein R-Spondin 2 promotes midbrain dopaminergic neurogenesis and differentiation. Stem Cell Rep. 2018;11:651-64.

32. Feinstein $Y$, Borrell V, Garcia C, Burstyn-Cohen T, Tzarfaty V, Frumkin A, Nose A, Okamoto H, Higashijima S, Soriano E, Klar A. F-spondin and mindin: two structurally and functionally related genes expressed in the hippocampus that promote outgrowth of embryonic hippocampal neurons. Development. 1999:126:3637-48.

33. Boyne LJ, Fischer I, Shea TB. Role of vimentin in early stages of neuritogenesis in cultured hippocampal neurons. Int J Dev Neurosci. 1996;14:739-48.

34. Quinones-Hinojosa A, Sanai N, Soriano-Navarro M, Gonzalez-Perez O, Mirzadeh Z, Gil-Perotin S, Romero-Rodriguez R, Berger MS, Garcia-Verdugo JM, Alvarez-Buylla A. Cellular composition and cytoarchitecture of the adult human subventricular zone: a niche of neural stem cells. J Comp Neurol. 2006:494:415-34.

35. Castorena KM, Peltier DC, Peng W, Miller DJ. Maturation-dependent responses of human neuronal cells to western equine encephalitis virus infection and type I interferons. Virology. 2008;372:208-20.

36. Ormel PR, Vieira de Sa $R$, van Bodegraven EJ, Karst $H$, Harschnitz $O$, MAM S, Johansen LE, van Dijk RE, Scheefhals N, Berdenis van Berlekom A, et al. Microglia innately develop within cerebral organoids. Nat Commun. 2018;9:4167.

37. Renner M, Lancaster MA, Bian S, Choi H, Ku T, Peer A, Chung K, Knoblich JA. Self-organized developmental patterning and differentiation in cerebral organoids. EMBO J. 2017:36:1316-29.

38. Qian X, Song $H$, Ming GL. Brain organoids: advances, applications and challenges. Development. 2019;146:dev166074. https://doi.org/10.1242/dev. 166074. Published 16 April 2019.

39. Qian X, Nguyen HN, Song MM, Hadiono C, Ogden SC, Hammack C, Yao B, Hamersky GR, Jacob F, Zhong C, et al. Brain-region-specific Organoids using mini-bioreactors for modeling ZIKV exposure. Cell. 2016;165:1238-54.

40. Trujillo CA, Gao R, Negraes PD, Gu J, Buchanan J, Preissl S, Wang A, Wu W Haddad GG, Chaim IA, et al. Complex oscillatory waves emerging from cortical organoids model early human brain network development. Cell Stem Cell. 2019;25:1-12.

41. Pleasure SJ, Page C, Lee VM. Pure, postmitotic, polarized human neurons derived from NTera 2 cells provide a system for expressing exogenous proteins in terminally differentiated neurons. J Neurosci. 1992;12:1802-15.

42. Dimou L, Gotz M. Glial cells as progenitors and stem cells: new roles in the healthy and diseased brain. Physiol Rev. 2014;94:709-37.

43. Catapano LA, Arnold MW, Perez FA, Macklis JD. Specific neurotrophic factors support the survival of cortical projection neurons at distinct stages of development. J Neurosci. 2001;21:8863-72. 
44. Luo MH, Hannemann H, Kulkarni AS, Schwartz PH, O'Dowd JM, Fortunato EA. Human cytomegalovirus infection causes premature and abnormal differentiation of human neural progenitor cells. J Virol. 2010;84:3528-41.

45. Morrey JD, Siddharthan V, Wang H, Hall JO, Skirpstunas RT, Olsen AL, Nordstrom JL, Koenig S, Johnson S, Diamond MS. West Nile virus-induced acute flaccid paralysis is prevented by monoclonal antibody treatment when administered after infection of spinal cord neurons. J Neuro-Oncol. 2008;14:152-63.

46. Oberhaus SM, Smith RL, Clayton GH, Dermody TS, Tyler KL. Reovirus infection and tissue injury in the mouse central nervous system are associated with apoptosis. J Virol. 1997;71:2100-6.

47. Tsunoda I, Kurtz Cl, Fujinami RS. Apoptosis in acute and chronic central nervous system disease induced by Theiler's murine encephalomyelitis virus. Virology. 1997;228:388-93.

48. van Marle G, Antony J, Ostermann H, Dunham C, Hunt T, Halliday W, Maingat F, Urbanowski MD, Hobman T, Peeling J, Power C. West Nile virusinduced neuroinflammation: glial infection and capsid protein-mediated neurovirulence. J Virol. 2007;81:10933-49.

49. Schoneboom BA, Catlin KM, Marty AM, Grieder FB. Inflammation is a component of neurodegeneration in response to Venezuelan equine encephalitis virus infection in mice. J Neuroimmunol. 2000;109:132-46.

50. Amor S, Puentes F, Baker D, van der Valk P. Inflammation in neurodegenerative diseases. Immunology. 2010;129:154-69.

51. Dekkers MP, Nikoletopoulou V, Barde YA. Cell biology in neuroscience: death of developing neurons: new insights and implications for connectivity. J Cell Biol. 2013;203:385-93.

52. Mukherjee P, Winkler CW, Taylor KG, Woods TA, Nair V, Khan BA, Peterson KE. SARM1, not MyD88, mediates TLR7/TLR9-induced apoptosis in neurons. J Immunol. 2015;195:4913-21.

53. Winkler CW, Taylor KG, Peterson KE. Location is everything: let-7b microRNA and TLR7 signaling results in a painful TRP. Sci Signal. 2014;7:pe14.

54. Lehmann SM, Kruger C, Park B, Derkow K, Rosenberger K, Baumgart J, Trimbuch T, Eom G, Hinz M, Kaul D, et al. An unconventional role for miRNA: let-7 activates toll-like receptor 7 and causes neurodegeneration. Nat Neurosci. 2012;15:827-35.

55. Farmer JR, Altschaefl KM, O'Shea KS, Miller DJ. Activation of the type I interferon pathway is enhanced in response to human neuronal differentiation. PLoS One. 2013;8:e58813.

56. Blakqori G, Delhaye S, Habjan M, Blair CD, Sanchez-Vargas I, Olson KE, Attarzadeh-Yazdi G, Fragkoudis R, Kohl A, Kalinke U, et al. La Crosse bunyavirus nonstructural protein NSs serves to suppress the type I interferon system of mammalian hosts. J Virol. 2007:81:4991-9.

57. Borsini A, Cattaneo A, Malpighi C, Thuret S, Harrison NA, Consortium MRCI Zunszain PA, Pariante CM. Interferon-alpha reduces human hippocampal neurogenesis and increases apoptosis via activation of distinct STAT1dependent mechanisms. Int J Neuropsychopharmacol. 2018;21:187-200.

58. Hirsch M, Knight J, Tobita M, Soltys J, Panitch H, Mao-Draayer Y. The effect of interferon-beta on mouse neural progenitor cell survival and differentiation. Biochem Biophys Res Commun. 2009;388:181-6.

\section{Publisher's Note}

Springer Nature remains neutral with regard to jurisdictional claims in published maps and institutional affiliations. 